\title{
PR55a regulatory subunit of PP2A inhibits the MOB1/LATS cascade and activates YAP in pancreatic cancer cells
}

Ashley L. Hein ${ }^{1}$, Nichole D. Brandquist ${ }^{1}$, Caroline Y. Ouellette ${ }^{1}$, Parthasarathy Seshacharyulu ${ }^{2}$, Charles A. Enke ${ }^{1}$, Michel M. Ouellette ${ }^{2,3}$, Surinder K. Batra ${ }^{2}$ and Ying Yan $\mathbb{B}^{1,2}$

\begin{abstract}
PP2A holoenzyme complexes are responsible for the majority of Ser/Thr phosphatase activities in human cells. Each PP2A consists of a catalytic subunit (C), a scaffold subunit (A), and a regulatory subunit (B). While the $A$ and $C$ subunits each exists only in two highly conserved isoforms, a large number of $B$ subunits share no homology, which determines PP2A substrate specificity and cellular localization. It is anticipated that different PP2A holoenzymes play distinct roles in cellular signaling networks, whereas PP2A has only generally been defined as a putative tumor suppressor, which is mostly based on the loss-of-function studies using pharmacological or biological inhibitors for the highly conserved A or C subunit of PP2A. Recent studies of specific pathways indicate that some PP2A complexes also possess tumor-promoting functions. We have previously reported an essential role of PR55a, a PP2A regulatory subunit, in the support of oncogenic phenotypes, including in vivo tumorigenicity/metastasis of pancreatic cancer cells. In this report, we have elucidated a novel role of PR55a-regulated PP2A in the activation of YAP oncoprotein, whose function is required for anchorage-independent growth during oncogenesis of solid tumors. Our data show two lines of YAP regulation by PR55a: (1) PR55a inhibits the MOB1-triggered autoactivation of LATS1/2 kinases, the core member of the Hippo pathway that inhibits YAP by inducing its proteasomal degradation and cytoplasmic retention and (2) PR55a directly interacts with and regulates YAP itself. Accordingly, PR55a is essential for YAP-promoted gene transcriptions, as well as for anchorage-independent growth, in which YAP plays a key role. In summary, current findings demonstrate a novel YAP activation mechanism based on the PR55a-regulated PP2A phosphatase.
\end{abstract}

\section{Introduction}

The PP2A (protein phosphatase 2A) family of heterotrimers accounts for the majority of serine/threonine phosphatase activities in human cells ${ }^{1,2}$. Each PP2A consists of one catalytic subunit $(\mathrm{C})$, one scaffolding subunit (A), and one regulatory subunit (B) ${ }^{1,2}$ (Fig. 1).

Correspondence: Surinder K. Batra (sbatra@unmc.edu) or Ying Yan (yyan@unmc.edu)

'Department of Radiation Oncology, University of Nebraska Medical Center, Omaha, NE, USA

${ }^{2}$ Department of Biochemistry and Molecular Biology, University of Nebraska Medical Center, Omaha, NE, USA

Full list of author information is available at the end of the article.

These authors contributed equally: Ashley L. Hein, Nichole D. Brandquist
While the $\mathrm{A}$ and $\mathrm{C}$ subunits each contain two highly conserved isoforms, a large number of the $B$ subunits are classified into four distinct subfamilies $\left(\mathrm{B}, \mathrm{B}^{\prime}, \mathrm{B}^{\prime \prime}\right.$, and $\left.\mathrm{B}^{\prime \prime \prime}\right)$ and share no homology. It is the $B$ subunit that determines the substrate specificities and cellular localizations of PP2 $\mathrm{A}^{1,3}$.

PP2A regulates diversified cellular functions, including proliferation, migration, and survival, whereas its role in oncogenesis remains poorly defined ${ }^{1,2,4-7}$. Currently, PP2A is defined as a putative tumor suppressor, mostly based on the loss-of-function analyses using inhibitors for the highly conserved $\mathrm{A} / \mathrm{C}$ subunits, such as okadaic acid (C-subunit inhibitor), short interfering RNA (siRNA)

\section{(c) The Author(s) 2019}

(c) (i) Open Access This article is licensed under a Creative Commons Attribution 4.0 International License, which permits use, sharing, adaptation, distribution and reproduction c. in any medium or format, as long as you give appropriate credit to the original author(s) and the source, provide a link to the Creative Commons license, and indicate if changes were made. The images or other third party material in this article are included in the article's Creative Commons license, unless indicated otherwise in a credit line to the material. If material is not included in the article's Creative Commons license and your intended use is not permitted by statutory regulation or exceeds the permitted use, you will need to obtain permission directly from the copyright holder. To view a copy of this license, visit http://creativecommons.org/licenses/by/4.0/. 


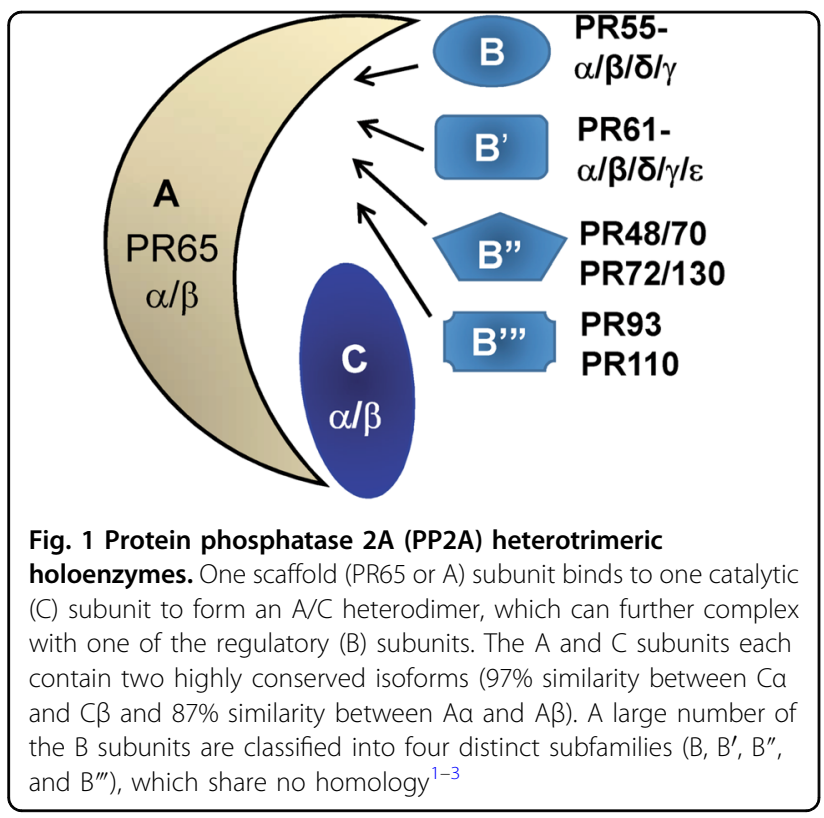

against the $\mathrm{A} / \mathrm{C}$ subunits, or viral oncoproteins displacing the B subunits (e.g., SV40 small-t) ${ }^{6,8,9}$. In addition, PP2AB subunits PR61 $(\alpha / \gamma)$ and PR72 act as tumor suppressors preventing oncogenic transformation ${ }^{10-13}$. In contrast, PP2A-B subunits PR130 and PR55 $\alpha$ function as tumorpromoters $^{1,2,14}$. While PR130 sustains EGF-mediated survival signaling and supports metastasis ${ }^{15,16}$, PR55 $\alpha$ activates the Raf/MEK/ERK oncogenic cascade by dephosphorylating KSR-S392 and Raf-S259/S295, and stabilizes $\beta$-catenin and c-Myc oncoproteins by dephosphorylating $\beta$-catenin-T41/S37/S33 and c-Myc-T58, respectively ${ }^{17-19}$. Furthermore, we have reported the role of PR55 $\alpha$ in the support of tumorigenicity and metastasis of pancreatic cancer cells ${ }^{20}$.

Yes-associated protein (YAP), a transcription coactivator, activates essential genes for tumorigenesis and metastasis of most solid tumors ${ }^{21-24}$. YAP is also required for KRAS-driven pancreatic tumorigenesis and compensates for KRAS-loss in the KRAS-addicted pancreatic cancer cells to produce malignant phenotypes ${ }^{25-27}$. YAP is inhibited by the Hippo tumor suppressor pathway that induces YAP phosphorylation at multiples sites, including YAP-S127 causing YAP cytoplasmic retention by 14-3-3, and YAP-S397 triggering YAP degradation by $\beta \operatorname{TrCP}-\mathrm{SCF}^{28}$.

The Hippo pathway primarily consists of MST1/2, MOB1, and LATS1/2. Upon activation, MST1/2 phosphorylates LATS1-T1079/LATS2-T1041, while it concurrently autophosphorylates itself between the catalytic-domain and the SARAH-domain to create a MOB1-docking site for inducing MOB1-T12/T35 phosphorylation $^{29-31}$, which subsequently triggers the autophosphorylation of LATS1-S909/LATS2-S872, leading to LATS1/2 autoactivation ${ }^{31}$.

The kinase roles in the Hippo pathway and YAP activation have been clearly elucidated, whereas the essential input of phosphatases in this regulation remains poorly understood. Previous studies implicate PP2A in the regulation of the Hippo pathway: while proteomics/RNAi screening show that dSTRIPAK-associated PP2A suppresses the Hippo pathway in Drosophila, PP2A inhibition by okadaic acid induces MOB1-phosphorylation in yeast cells and MST1/2-phosphorylation in HeLa cervical cancer cells ${ }^{32-34}$. The current study reveals a critical role of PR55 $\alpha$ in the inhibition of the MOB1/LATS autoactivation loop and activation of YAP in pancreatic normal and cancer cells.

\section{Results}

PR55a supports the activation of YAP in pancreatic cancer cells

We have shown that PR55 $\alpha$ supports anchorageindependent growth and tumorigenicity of pancreatic cancer cells ${ }^{20}$, which is also the best-known function of YAP in cancer ${ }^{21,24}$. Immunoblotting detected a marked increase in YAP level in human pancreatic cancer cells relative to the HPNE human normal pancreatic cells immortalized with telomerase ${ }^{35}$ (Fig. 2). In contrast, YAPS127 phosphorylation, which induces YAP cytoplasmic retention by 14-3-3, is only moderately increased in pancreatic cancer cells compared with HPNE.

YAP inhibition by the LATS1/2 kinases is primarily via two mechanisms: phosphorylation of YAP-S397 leading to proteasomal degradation by $\beta-\operatorname{TrCP}$ and phosphorylation of YAP-S127 resulting in 14-3-3 cytoplasmic retention $^{36}$. Therefore, we examined the impact of PR55 $\alpha$ on YAP activation in pancreatic cancer cells using a series of Doxycycline (Dox)-inducible PR55 $\alpha$ shRNAs. As shown in Fig. 3a, PR55 $\alpha$-knockdown by short-hairpin RNAs (shRNAs) markedly decreased PR55 $\alpha$ level in pancreatic cancer cells (CD18/HPAF and AsPC-1) compared with parental and Control-shRNAtransduced cells. Consequently, YAP level was largely reduced following PR55 $\alpha$-knockdown with a concomitant increase in YAP-S127 phosphorylation in the cells. On the other hand, the steady-state level of YAPS397 phosphorylation was not particularly increased following PR55 $\alpha$-knockdown. Since YAP-S397 phosphorylation is specifically linked to YAP proteasomal degradation and cannot accumulate in the cells ${ }^{37}$, this outcome was anticipated. Collectively, these results suggest a role of PR55 $\alpha$ in the maintenance of YAP protein and dephosphorylation of YAP-S127 in pancreatic cancer cells. 


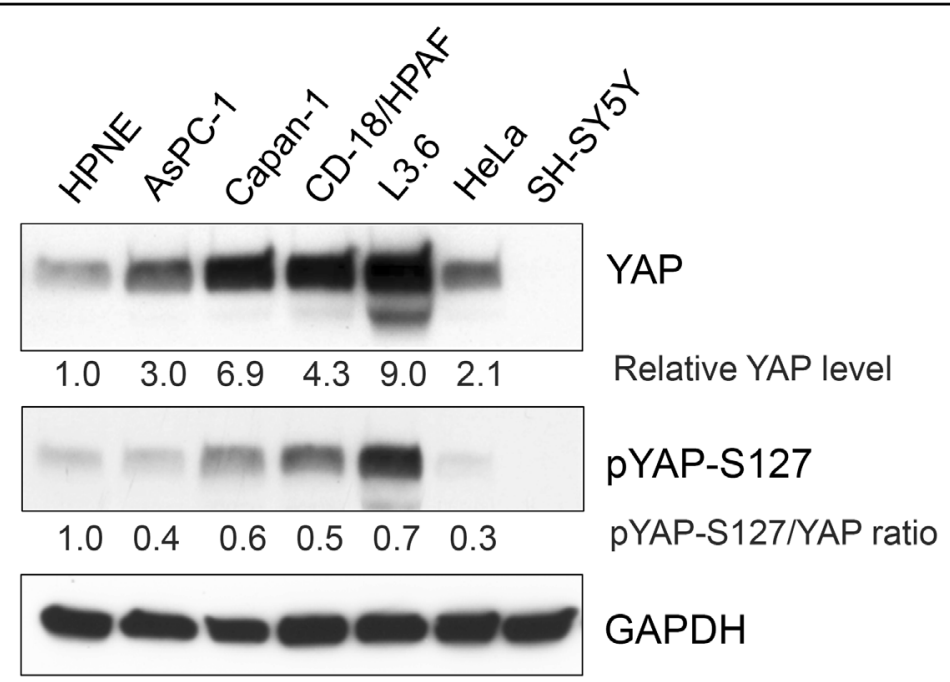

Fig. 2 Analysis of YAP protein level and YAP-S127 phosphorylation in normal and malignant human pancreatic cells. YAP protein level is markedly increased in the human pancreatic cancer cells (AsPC-1, Capan-1, CD18/HPAF, and L3.6) compared with human pancreatic ductal cells (HPNE), as determined by immunoblotting. HeLa human cervical cancer cells and SH-SY5Y human neuroblastoma cells serve as a positive and negative control, respectively, for YAP protein expression. GAPDH in the lysates was measured as internal controls. The protein levels of YAP, pYAPS127, and GAPDH were quantified using ImageJ software. Relative YAP and pYAP-S127 levels in the samples were normalized with GAPDH levels and the ratio of PYAP-S127/YAP determined

The effects of PR55a on the Hippo tumor suppressor pathway in pancreatic cancer cells

The Hippo pathway negatively regulates YAP level and activity. Therefore, we examined the effects of PR55 $\alpha$ on the core members of this pathway (MST1/2, MOB1, and LATS1/2) in pancreatic cancer cells using Dox-inducible shRNAs.

We tested the effect of PR55 $\alpha$ on the MST/LATS cascade in CD18/HPAF and AsPC-1 cells. Unexpectedly, PR55 $\alpha$-knockdown by shRNA resulted in decreases in MST1/2 levels and phosphorylation, along with the phosphorylation of its downstream targets LATS1-T1079/ LATS2-T1041 (Fig. 3a).

Next, we analyzed the role of PR55 $\alpha$ in the MOB1triggered autophosphorylation of LATS1-S909/LATS2S872, the key step leading to the LATS1/2 autoactivation loop ${ }^{37}$. In both CD18/HPAF and AsPC-1 cells, knockdown of PR55 $\alpha$ induced the phosphorylation of MOB1T35 and LATS1-S909/LATS2-S872 (MOB1 downstream targets), which indicates activation of the MOB1/LATS autoactivation loop ${ }^{28,38}$ (Fig. 3a). While LATS1 protein level remained unchanged, LATS2 protein was increased following PR55 $\alpha$-knockdown in CD18/HPAF cells. However, this effect was not detected in AsPC-1 cells. This difference probably attributes to cell-type specificity, as CD18/HPAF cells originated from metastatic liver lesions, while AsPC-1 cells were isolated from ascites ${ }^{39}$.

We validated the impact of PR55 $\alpha$ on the MOB/LATS/ YAP cascade with a time-course study. Following PR55 $\alpha$ knockdown by shRNA in CD18/HPAF cells, there was a time-dependent decrease in YAP level with a concurrent increase in YAP-S127 phosphorylation (Fig. 3b). Consistently, these changes in YAP were tightly associated with an increase in phosphorylation of both LATS1-S909/ LATS2-S872 and MOB1-T35, an increase of protein level of LATS2, and the diminution of LATS1-T1079/LATS2T1041 phosphorylation (Fig. 3b).

Since LATS2 was reported to be regulated by proteasomal degradation $^{40}$, we evaluated the effect of PR55 $\alpha$ on LATS2 protein stability using protein synthesis inhibitor cycloheximide (CHX), as described in our study ${ }^{41}$. The analysis indicated that LATS2 protein half-life is $\sim 3.2 \mathrm{~h}$ in CD18/HPAF cells, while it elongated to $\sim 6.7 \mathrm{~h}$ after the knockdown of PR55 $\alpha$ (Fig. 3c).

Collectively, these results suggest an essential role for PR55 $\alpha$ in the inhibition of the MOB1/LATS cascade that directly prevents YAP activation.

\section{Ectopic PR55a expression induces YAP activation in normal human pancreatic ductal cells}

To define the role of PR55 $\alpha$ in normal pancreatic cells, we constructed the pREV-TRE-PR55 $\alpha$ retroviral vector expressing Dox-inducible PR55 $\alpha$, which was further stably introduced into HPNE normal pancreatic ductal cells (Fig. 4a). Following $48 \mathrm{~h}$ induction with increasing doses of Dox, a marked increase in PR55 $\alpha$ protein was detected in the pREV-TRE-PR55 $\alpha$-transduced cells but not in the control-vector-transduced cells (Fig. 4b). Associated with the PR55 $\alpha$ induction were a concomitant increase of YAP protein levels and a simultaneous diminution of YAP- 
a
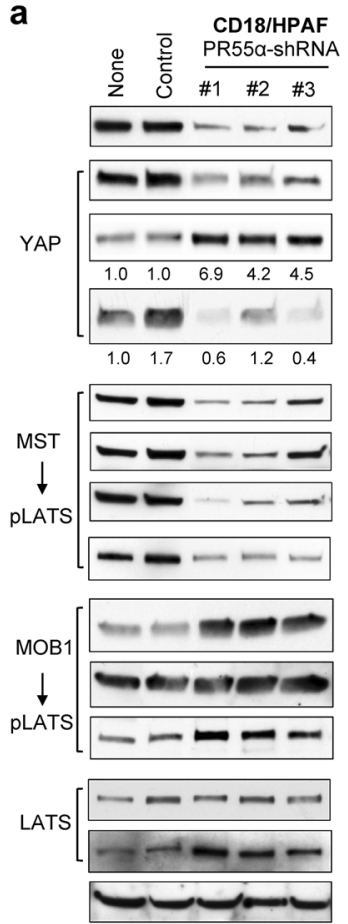

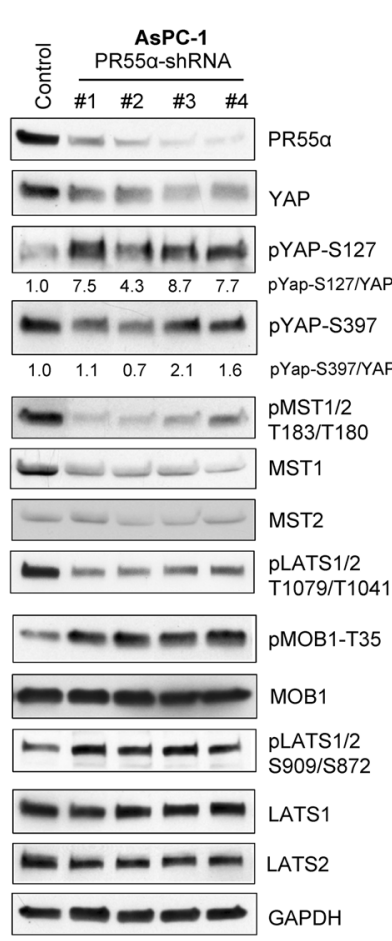

b

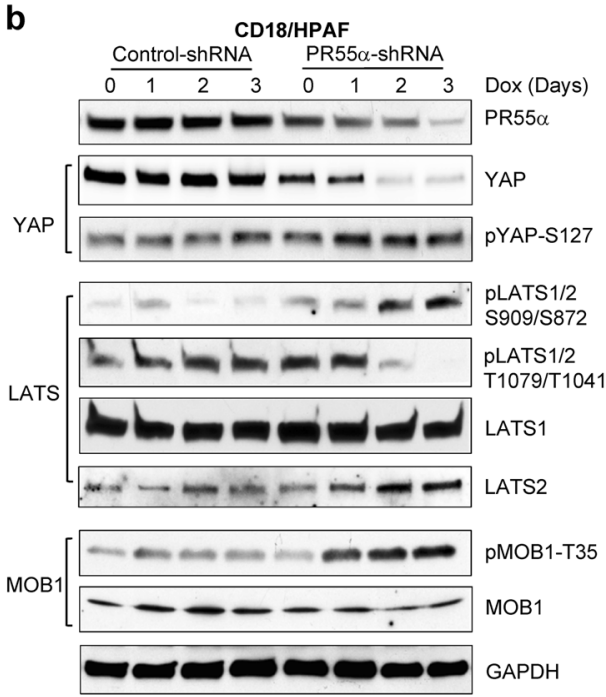

C

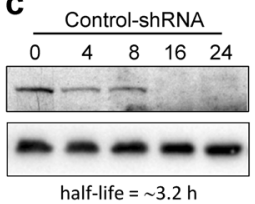

Fig. 3 PR55a-knockdown by shRNA inhibits YAP and activates the MOB1-LATS1/2 cascade in pancreatic cancer cells. a CD18/HPAF and AsPC-1 cells were stably transduced with Dox-inducible shRNAs targeting various regions of PR55a or, as a control, with nontargeting shRNA. After incubation with Dox $(2 \mu \mathrm{g} / \mathrm{ml})$ for 3 days, the cell lysates $(100 \mu \mathrm{g})$ were analyzed by immunoblotting for the indicated protein levels and/or phosphorylation. GAPDH served as an internal protein expression control. $\mathbf{b}$ Validation of the effects of PR55a on MOB1/LATS/YAP cascade in pancreatic cancer cells. shRNA-transduced CD18/HPAF cells were incubated with Dox $(2 \mu \mathrm{g} / \mathrm{ml})$ for the days indicated and analyzed for PR55a expression and the levels/phosphorylation of MOB1, LATS1/2, and YAP. GAPDH serves as an internal control. c To test the effect of PR55a on LATS2 protein stability, shRNA-transduced CD18/HPAF cells were incubated in medium containing cycloheximide (CHX, $15 \mu \mathrm{g} / \mathrm{ml})$ to halt protein synthesis for the indicated hours. Whole-cell extracts were analyzed for LATS2 and GAPDH protein levels by immunoblotting. The protein levels of LATS2 and GAPDH were quantified using ImageJ software; relative LATS2 levels were normalized with GAPDH levels in the samples and protein half-life determined using SigmaPlot (version 11.2) analytical program

S127/S397 phosphorylation (Fig. 4b). In contrast, controlvector-transduced cells receiving the same treatment showed little effect on either PR55 $\alpha$ expression or on YAP protein level/phosphorylation.

\section{Effects of ectopic PR55a on MOB1/LATS cascade in normal pancreatic ductal cells}

We next examined the effect of PR55 $\alpha$ on LATS1/2 phosphorylation and levels in HPNE cells. Ectopic PR55 $\alpha$ expression in HPNE cells resulted in a marked decrease in LATS1-S909/LATS2-S872 phosphorylation along with a moderate increase in LATS1-T1079/LATS2-T1041 phosphorylation (Fig. 4b). Furthermore, LATS2 protein level was reduced in HPNE cells upon PR55 $\alpha$ overexpression, while the effect is absent in the control cells (Fig. 4b). With a time-course study, we verified this effect of PR55 $\alpha$. Figure 4c shows that, following PR55 $\alpha$ induction by Dox in HPNE cells, YAP protein level was increased, along with concomitant reductions in phosphorylation of YAP-S127, MOB1-T35, and LATS1-S909/
LATS2-S872, and a decrease in LATS2 protein level. Immunoblotting also detected an increase in MST1/2 phosphorylation and level and LATS1-T1079/LATS2T1041 phosphorylation (MST1/2 substrates) in HPNE cells.

Collectively, the results from both normal and malignant cells suggest a role of PR55 $\alpha$ in YAP activation that involves the suppression of the MOB1/LATS autoactivation loop, leading to YAP phosphorylation/inhibition. Furthermore, this role of PR55 $\alpha$ apparently does not require the MST/LATS cascade, since its activity increases in response to PR55 $\alpha$ overexpression. This may implicate a feedback loop activation by PR55 $\alpha$.

\section{PR55a supports YAP protein stability}

One of the primary mechanisms by which the Hippo pathway inhibits YAP is to induce its proteasomal degradation ${ }^{42}$. We, therefore, assessed the effect of PR55 $\alpha$ on YAP protein stability in both the cytoplasm and nuclei of CD18/HPAF cells using $\alpha$-tubulin and Lamin $\mathrm{A} / \mathrm{C}$ as 
a

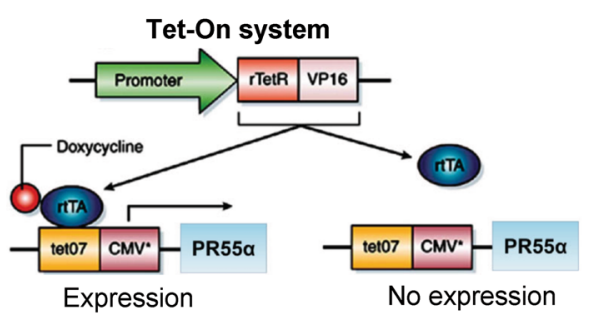

b
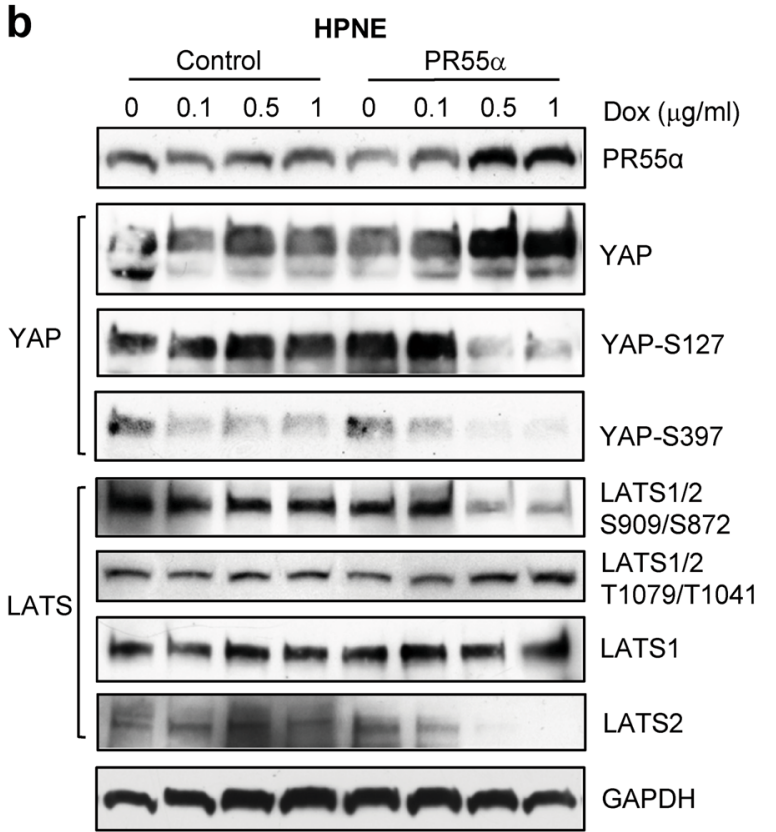

C

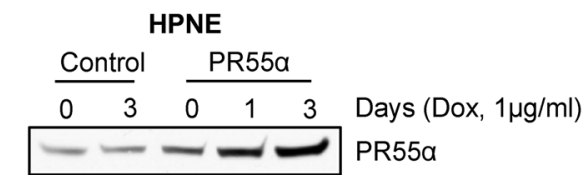
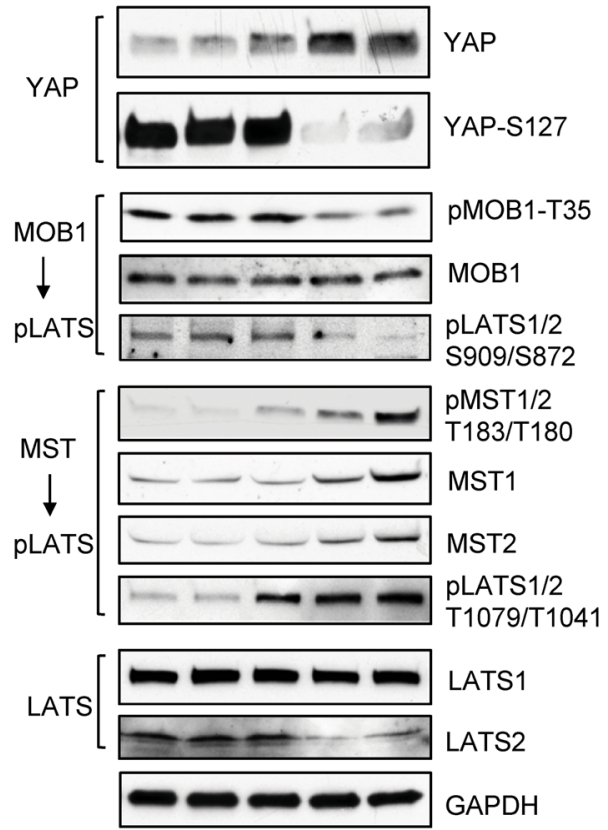

Fig. 4 Ectopic PR55a expression in human normal pancreatic ductal cells (HPNE) activates YAP and inhibits the MOB1-LATS1/2 cascade. a Dox-inducible retroviral vector expressing PR55a (pRevTRE-PR55a) was constructed and retrovirus was produced as described in the "Materials and methods" section. Subsequently, HPNE cells were transduced with both pRevTet-On (Clontech) that expresses rtTA and pRevTRE-PR55a (or pRevTREControl) and selected for resistant cells to both G418 $(400 \mu \mathrm{g} / \mathrm{ml})$ and Hygromycin B $(200 \mu \mathrm{g} / \mathrm{ml})$. Diagram demonstrates the Tet-inducible retroviral vector (pRevTRE-PR55a) expressing PR55a. $\mathbf{b}$ The transduced cells were induced for ectopic PR55a expression by incubation with increasing doses of Dox $(1 \mu \mathrm{g} / \mathrm{ml})$ for 3 days and the effect of PR55a on the phosphorylation and level of YAP and LATS1/2 analyzed by immunoblotting. GAPDH served as an internal control. c Control- and PR55a-transduced HPNE cells were incubated with Dox (1 $\mu \mathrm{g} / \mathrm{ml})$ for the indicated days and analyzed for the effect of PR55a on YAP and the Hippo pathway (MST1/2, MOB1, and LATS1/2), by analyzing their phosphorylation and levels by immunoblotting. GAPDH served as an internal control

cytoplasmic and nuclear markers, respectively ${ }^{43}$. Immunoblotting showed that PR55 $\alpha$ is ubiquitously expressed in both cytoplasm and nuclei of the cells and its level increases following proteasomal inhibition by MG132 (Fig. 5a). To test whether the decrease of YAP in PR55 $\alpha$ knockdown cells was due to proteasomal degradation, MG132 was used to block proteasome activity and assessed for its effect on YAP level. As shown in Fig. 5b, MG132 treatment of the PR55 $\alpha$-knockdown cells resulted in an induction of both cytoplasmic and nuclear YAP, while MG132 treatment of Control-shRNA knockdown cells caused a decrease in cytoplasmic YAP level and only a subtle increase in the nuclear YAP. This suggests that
PR55 $\alpha$ inhibits YAP proteasomal degradation in CD18/ HPAF cells.

We tested the effect of PR55 $\alpha$ on YAP protein half-life in the cytoplasm and nuclei of CD18/HPAF cells, which were treated with CHX to block protein synthesis, and analyzed for YAP protein decay over time by western blot. The results show that YAP protein half-life was $\sim 15.5$ and $20.6 \mathrm{~h}$ in the cytoplasm and nuclei of the control cells, respectively, whereas it was only $\sim 8$ and $\sim 8.7 \mathrm{~h}$ in the cytoplasm and nuclei of the PR55 $\alpha$-knockdown cells, respectively (Fig. 5c). These findings suggest that PR55 $\alpha$ supports YAP protein stability via a mechanism that involves the inhibition of the proteasomal degradation of YAP. 


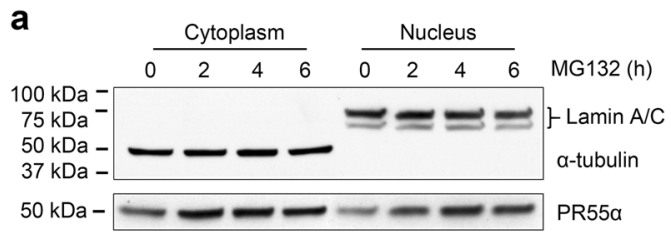

b

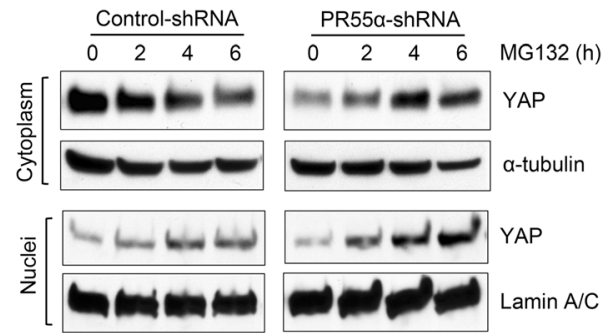

C
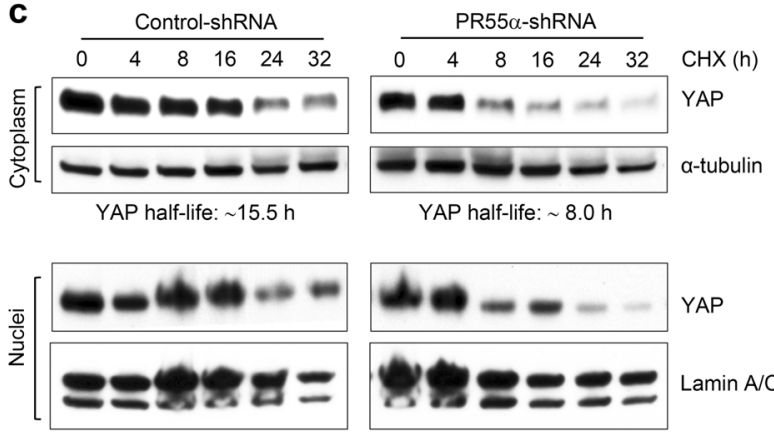

YAP half-life: $\sim 20.6 \mathrm{~h}$

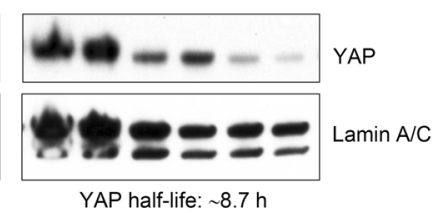

Fig. 5 PR55a is essential for maintaining YAP stability. Cytoplasmic and nuclear extracts were isolated using the NE-PER ${ }^{\text {TM }}$ Nuclear and Cytoplasmic Extraction Reagents (Fisher Scientific) and analyzed for YAP protein expression. a a-tubulin and Lamin A/C were used as the cytoplasmic and nuclear markers, respectively. PR55a in cytoplasm and nucleus was analyzed by Western blot analysis. $\mathbf{b}$ To inhibit cellular proteasome activity, CD18/HPAF cells transduced with Control-shRNA or PR55a-shRNA were treated with MG132 (25 $\mu \mathrm{M})$ for the indicated hours. Cytoplasmic and nuclear extracts were isolated from the treated cells and analyzed for YAP expression by immunoblotting. a-tubulin and Lamin A/C served as the internal controls for cytoplasmic and nuclear extract, respectively. c To test the effect of PR55a on YAP protein stability, Control-/PR55a-shRNA-transduced CD18/HPAF cells were treated with CHX $(15 \mu \mathrm{g} / \mathrm{ml})$ to halt protein synthesis for the indicated hours, cytoplasmic and nuclear extracts were isolated from the treated cells, and YAP protein levels analyzed by immunoblotting. a-tubulin and Lamin A/C served as internal controls for cytoplasmic and nuclear extract, respectively

\section{Interaction of PR55a and YAP in pancreatic normal and cancer cells}

By reciprocal co-immunoprecipitation assay described previously ${ }^{41}$, we examined the interaction of PR55 $\alpha$ and YAP in CD18/HPAF cells. As a control, immunoprecipitation with nonimmunized IgG was included in the study. Immunoblotting revealed the presence of both PR55 $\alpha$ and YAP, along with PP2A-A and PP2A-C subunits in the immunoprecipitates obtained either with anti-PR $55 \alpha$ or anti-YAP antibody (Fig. 6a). Furthermore, relative to control cells, PR55 $\alpha$-knockdown cells displayed a lesser amount of PR55 $\alpha$, PP2A-C, and PP2A-A subunits in the immunoprecipitates obtained with antiPR55 $\alpha$ or anti-YAP antibody (Fig. 6a). Unexpectedly, relative to control cells, a higher level of YAP was detected in the anti-PR55 $\alpha$ immunoprecipitates obtained from PR55 $\alpha$-knockdown cells, which is inconsistent with a lesser amount of YAP revealed in the anti-YAP immunoprecipitates obtained from PR55 $\alpha$-knockdown cells relative to control cells (Fig. 6a).

To determine whether the high YAP level present in the anti-PR55 $\alpha$ immunoprecipitates from PR55 $\alpha$-knockdown cells could be attributed to YAP hyperphosphorylation, the anti-PR55 $\alpha$ immunoprecipitates from control and PR55 $\alpha$-knockdown cells were treated with/without shrimp alkaline phosphatase (SAP) and analyzed for PR55 $\alpha$ and p-YAP/YAP levels. As shown in Fig. 6b, the high YAP level in the anti-PR55 $\alpha$ immunoprecipitates from PR55 $\alpha$-knockdown cells was markedly diminished after SAP treatment (YAP, lane 4 vs. 2). Immunoblotting of YAP-Ser127 phosphorylation confirmed the effectiveness of SAP in YAP dephosphorylation (YAP-S127, lane 4 vs. 2).

We compared the interaction of PR55 $\alpha$ with YAP [wildtype (WT)] versus YAP(5SA) (constitutive active mutant), in which the LATS phosphorylation sites (S61/S109/ S127/S164/S397) were mutated to alanine ${ }^{38,42}$. CD18/ HPAF cells were stably transduced with Flag-YAP(WT) or Flag-YAP(5SA) and immumonoprecitated with antiPR55 $\alpha$ antibody. As shown in Fig. 6c, anti-Flag antibody detected the presence of Flag-YAP and Flag-YAP(5SA) in the anti-PR55 $\alpha$ immunoprecipitates obtained from their respective lysate, whereas the Flag-YAP(WT) level was 28-fold higher than Flag-YAP(5SA). This result implicates a direct interaction of PR55 $\alpha$ and YAP and further supports that PR55 $\alpha$ expresses a higher affinity toward phosphorylated YAP.

Using immunofluorescence (IF) confocal microscopy, we analyzed the intracellular level, distribution, and colocalization of PR $55 \alpha$ and YAP. The results show the detection of PR55 $\alpha$ and YAP in both the cytoplasm and nucleus of CD18/HPAF cells, with PR55 $\alpha$ slightly more concentrated in the cytoplasm and YAP slightly more in the nucleus (shControl, Fig. 6d and Supplementary Fig. S1a). In the PR55 $\alpha$-knockdown cells, both PR55 $\alpha$ and YAP levels were markedly reduced and the residual YAP 


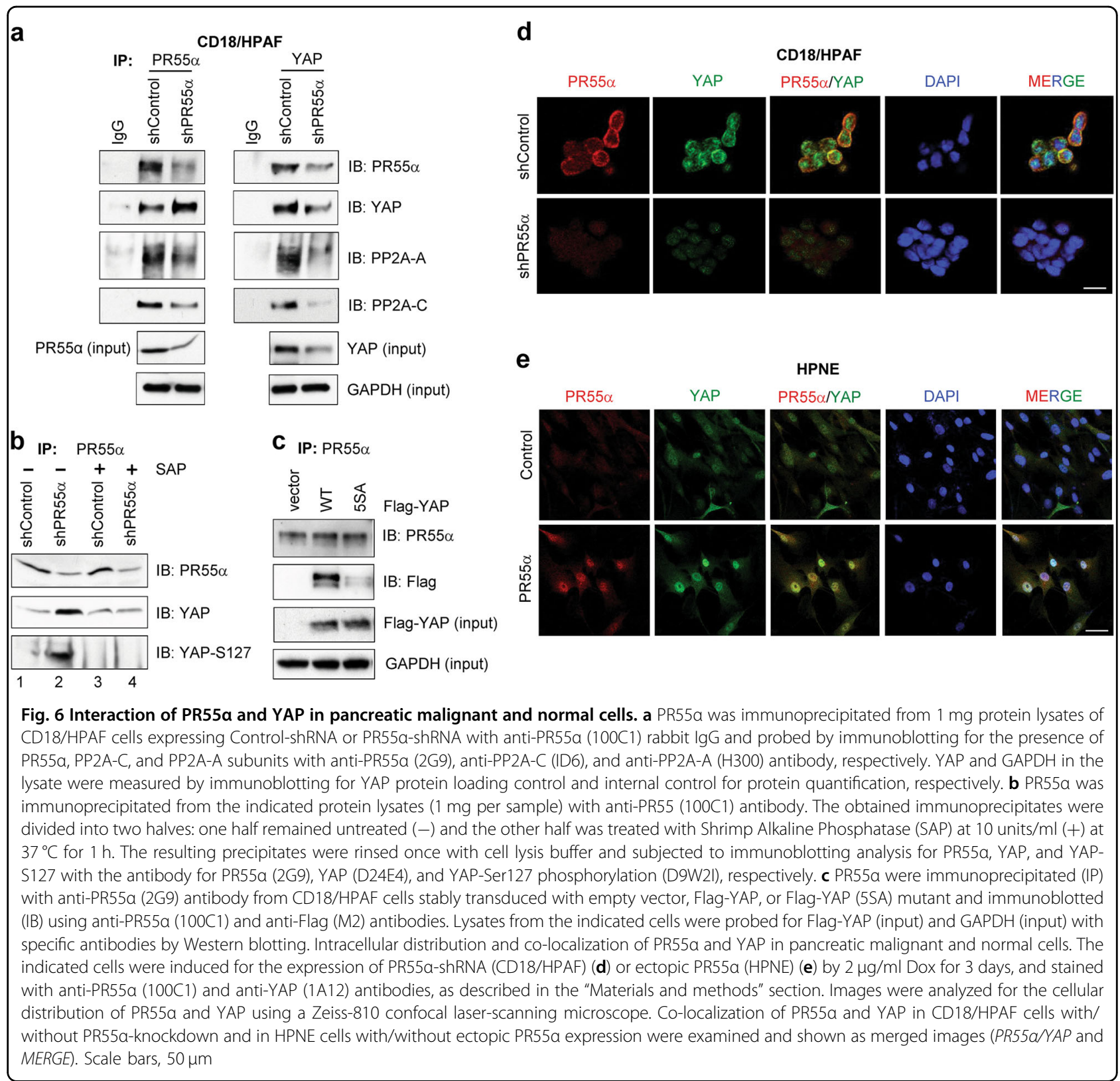

was now mainly present in the nucleus $(\operatorname{sh} P R 55 \alpha$, Fig. 6d, and Supplementary Fig. S1a).

We next analyzed the intracellular distribution of PR55 $\alpha$ and YAP in normal HPNE cells. Both PR55 $\alpha$ and YAP were also detected in both the cytoplasm and nucleus of the cells (Fig. 6e and Supplementary Fig. S1b). Upon ectopic PR55 $\alpha$ expression, YAP and PR $55 \alpha$ protein levels were concurrently increased in the cells and the additional amounts of the proteins were predominantly detected in the nuclei.

Co-localization studies revealed that, in both CD18/ HPAF and HPNE cells, there was a significantly greater co-localization of PR55 $\alpha$ and YAP in the PR55 $\alpha$-high cells (CD18/HPAF-shControl and HPNE-PR55 $\alpha$ ) compared with their respective isogeneic PR55 $\alpha$-low cells (CD18/ HPAF-shPR55 $\alpha$ and HPNE-PR55 $\alpha$ ) (Fig. 6d-e and Supplementary Fig. S1c).

Collectively, these results suggest a physical interaction and functional relationship of PR55 $\alpha$ and YAP in pancreatic cancer and normal cells.

\section{Effect of MOB1 on the PR55a-promoted YAP activation}

MOB1-triggered LATS1-S909/LATS2-S872 autophosphorylation is the key event resulting in YAP 
inhibition $^{38,44}$. The results in Figs. 3-4 demonstrate that PR55 $\alpha$-promoted YAP activation in both malignant and normal cells is inversely associated with the activity of the MOB1/LATS axis. We, therefore, probed the role of MOB1 in YAP activation by PR55 $\alpha$ using siRNA. MOB1 exists in two isoforms, MOB1A and MOB1B, which share $95 \%$ protein sequence identity and are functionally redundant ${ }^{44}$. Since there is no antibody available to distinguish MOB1A and MOB1B, we analyzed their expressions by RT-PCR in a panel of pancreatic normal and cancer cells, which showed that MOB1A mRNA level is 5-15 fold higher than MOB1B in these cells (Supplementary Fig 2a, b). This result was confirmed by siRNAknockdown studies, which showed that MOB1A-siRNA but not MOB1B-siRNA effectively reduced the total MOB1 protein level in the cells (Supplementary Fig 2c).

With MOB1A-siRNA, we assessed the role of MOB1 in the regulation of LATS1/2 and YAP by PR55 $\alpha$. MOB1knockdown in HPNE-Control cells resulted in a decrease of LATS1-S909/LATS2-S872 phosphorylation and a concurrent increase in YAP protein level, as shown in Fig. 7a (lane 2 vs. 1, Bar graph). Similarly, MOB1knockdown in HPNE-PR55 $\alpha$ cells caused a marginal decrease in the already low LATS1-S909/LAT2-S872 phosphorylation and increase of YAP level compared with the HPNE-PR55 $\alpha$ cells with control-knockdown (Fig. 7a, lane 4 vs. 3, Bar graph). However, MOB1-knockdown in HPNE cells produced no effect on YAP-S127 phosphorylation or LATS2 protein levels, which are negatively affected only by PR55 $\alpha$ level.

We next evaluated the effect of MOB1 on the regulation of LATS and YAP by PR55 $\alpha$ in malignant CD18/HPAF cells. While MOB1-knockdown by siRNA had very little effect on the low level of LATS1-S909/LATS2-S872 phosphorylation in CD18/HPAF cells, it markedly diminished the induction of LATS1-S909/LATS2-S872 phosphorylation caused by the PR55 $\alpha$-knockdown in CD18/HPAF cells (Fig. 7a, right panel, bar graph). In both control- and PR55 $\alpha$-shRNA-transduced CD18/HPAF cells, MOB1-knockdown by siRNA resulted in a subtle increase of YAP protein level but had little effect on YAPS127 phosphorylation, or LATS2 protein level (Fig. 7a, lane 6 vs. 5 and lane 8 vs. 7), all of which were significantly affected by the level of PR55 $\alpha$, (Fig. 7a, right panel, lanes 7-8 vs. lanes 5-6).

These results indicate that PR55 $\alpha$ suppresses the activation of MOB1/LATS cascade, while MOB1-inhibition cannot fully compensate for the loss of PR55 $\alpha$ to restore YAP activation, suggesting that PR55 $\alpha$ holds a dominant control on the magnitude of YAP activation.

\section{Effect of LATS1/2 in the PR55a-promoted YAP activation}

We investigated the role of LATS1/2 in the activation of YAP by $\mathrm{PR} 55 \alpha$ in CD18/HPAF and AsPC- 1 pancreatic cancer cells. In control-shRNA-transduced cells, knockdown of LATS1 and/or LATS2 by siRNA had only subtle effects on YAP phosphorylation and level in the cells (Fig. 7b, Control-shRNA). In PR55 $\alpha$-shRNA-transduced cells, knockdown of LATS1 or LATS2 alone by siRNA resulted in 1.6-3 fold increases in YAP protein levels relative to control cells (Fig. 7b, YAP, lanes 6-7 vs. lane 5). However, inhibition of both LATS1 and LATS2 by siRNA in the PR55 $\alpha$-knockdown cells resulted in a subtle, if any, decrease in YAP level compared with control cells (Fig. 7b, YAP, lane 8 vs. 5). Thus, in the PR55 $\alpha$-high (Control-shRNA) cells, manipulation of LATS1/2 levels apparently produced little effect on YAP level, whereas knockdown of either LATS1 or LATS2 in the PR55 $\alpha$-low (PR55 $\alpha$-shRNA) cells resulted in increases in YAP levels (Fig. 7b).

We also analyzed the effect of LATS1/2 on YAP-S127 phosphorylation in the presence/absence of PR55 $\alpha$ knockdown in pancreatic cancer cells. As shown in Fig. 7b, knockdown of LATS1 and/or LATS2 by siRNA had little effect on YAP-S127 phosphorylation in ControlshRNA-transduced cells, while it resulted in decreases in YAP-S127 phosphorylation in PR55 $\alpha$-shRNA-transduced cells. Furthermore, while knockdown of both LATS1 and LATS2 displayed an additive effect on inhibition of YAPS127 phosphorylation in CD18/HPAF cells, this effect was not observed in AsPC-1 cells. These results suggest that PR55 $\alpha$ plays a domainant role in the negative regulation of YAP-S127 phosphorylation.

\section{PR55a enhances YAP-targeted gene transcriptions and anchorage-independent growth}

To evaluate the biological significance of PR55 $\alpha$ in promoting YAP activation, we analyzed the effect of PR55 $\alpha$ on YAP-targeted gene expressions in HPNE (normal) and CD18/HPAF (malignant) cells. Real-time (RT)-PCR analyses revealed that PR55 $\alpha$ expression was positively associated with YAP-activated transcriptions of ANKRD1, CTGF, CYR61, and Survivin ${ }^{37}$ in both HPNE and CD18/HPAF cells (Fig. 8a). Thus, ectopic PR55 $\alpha$ expression in HPNE cells resulted in 8-10 fold increases in mRNA expressions of the YAP targets compared with control cells (red bars), while PR55 $\alpha$-knockdown by shRNA in CD18/HPAF cells caused a 4-6-fold reduction in mRNA levels of the YAP targets relative to the controlshRNA-transduced cells (black bars). These functional data confirm the role of PR55 $\alpha$ in the promotion of YAP activation.

Promoting anchorage independence is the predominant role of YAP in oncogenesis ${ }^{45-47}$. YAP alone has been shown to induce anchorage-independent growth of HPNE normal cells by the soft-agar assay ${ }^{22}$. Therefore, we tested the effect of PR55 $\alpha$ on anchorage-independent growth using the soft-agar assay ${ }^{48}$. The results in Fig. $8 \mathrm{~b}$ show that, following Dox-induced ectopic PR55 $\alpha$ expression, 


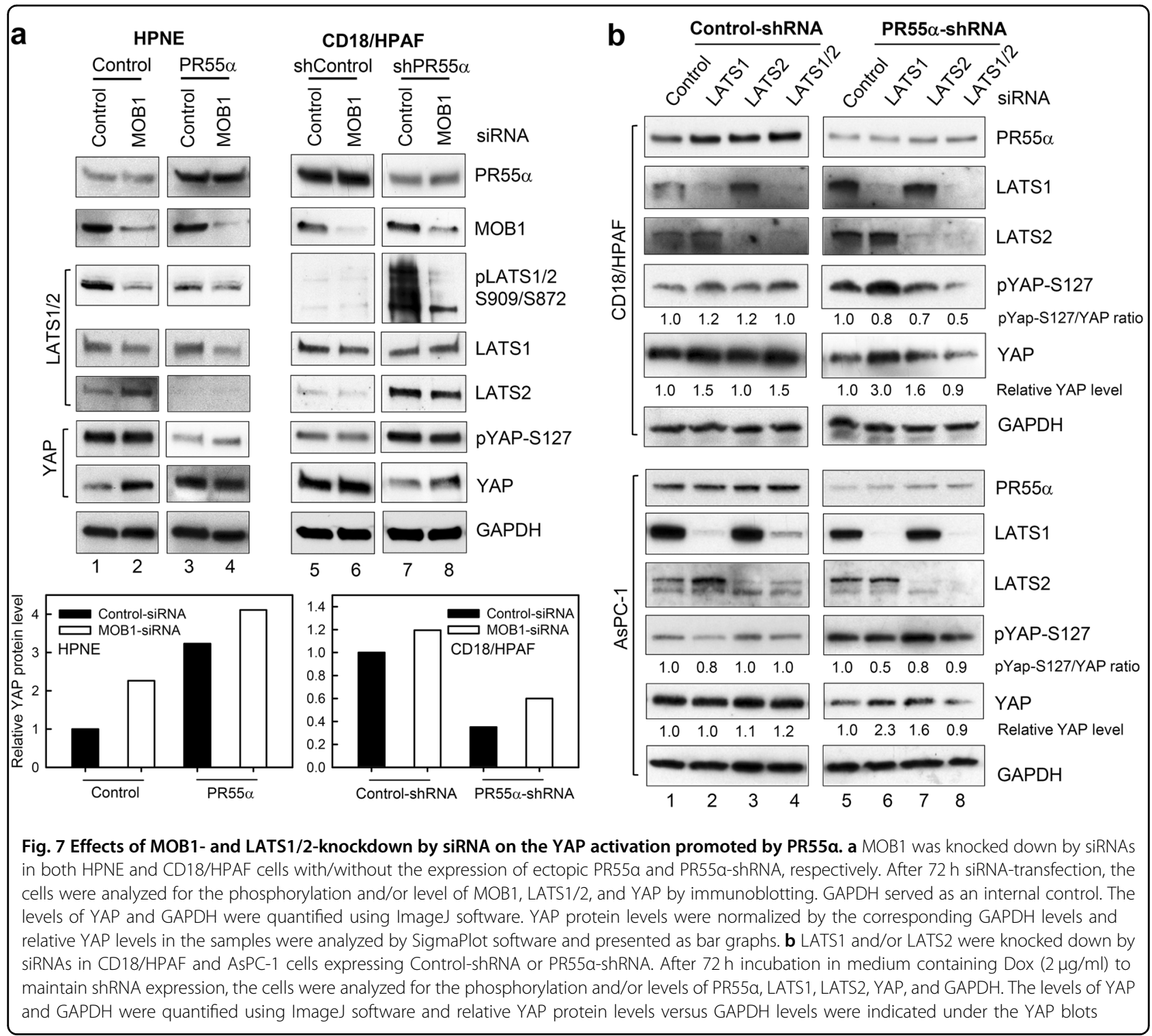

there was a significant induction of the proliferation of HPNE normal cells in soft-agar, indicative of anchorageindependent growth. Conversely, PR55 $\alpha$-knockdown by shRNA abrogated the clonogenicity of pancreatic cancer cells in soft-agar, indicative of loss of anchorage independence (Fig. 8c). These results support a critical role of $\mathrm{PR} 55 \alpha$ in the positive regulation of YAP oncogenic function.

In summary, the results of the current study (Figs. 2-8) reveal a novel mechanistic role of PR55 $\alpha$ regulated PP2A in the activation of YAP oncoprotein. Figure 9 outlines the findings of this investigation, which indicates that PR55 $\alpha$ specifically suppresses the MOB1-mediated LATS autophosphorylation/activation, which would otherwise promote YAP proteasomal degradation by $\beta-\operatorname{TrCP}$ and cytoplasmic retention by $14-3-3^{37}$. Furthermore, PR55 $\alpha$ also exhibited a Hippo pathway-independent role in YAP activation, as siRNA-knockdown of either MOB1 or LATS1/2 did not compensate completely for the effect of PR55 $\alpha$-loss or PR55 $\alpha$-overexpression on YAP activation in both normal and malignant pancreatic cells (see Fig. 7), which suggests a regulation of YAP activation directly by PR55 $\alpha$ or by another unknown mechanism regulated by PR55 $\alpha$.

\section{Discussion}

PP2A has been suggested in the regulation of the Hippo pathway and YAP activation ${ }^{32-34}$, while the specific PP2A holoenzyme(s) involved were not identified. We recently identified the PR55 $\alpha$ regulatory subunit of PP2A in the support of anchorage independence and tumorigenicity of pancreatic cancer cells, which coincidentally is the main 


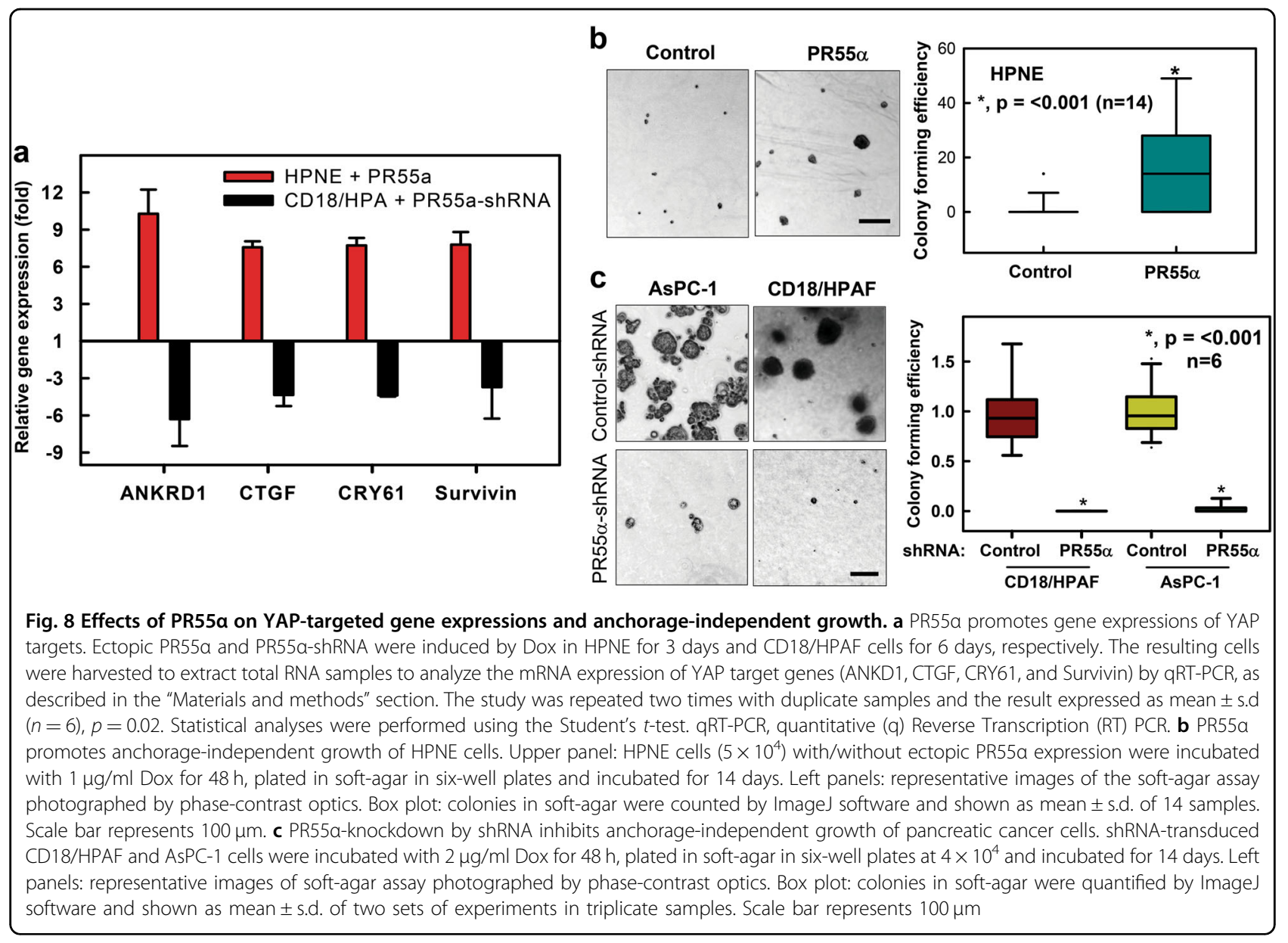

function of $\mathrm{YAP}^{20,49}$. Thus, we investigated the role of PR55 $\alpha$ in the regulation of the Hippo pathway and YAP activation in pancreatic cancer cells.

The results in this report elucidate a novel YAP activation mechanism based on the PR55 $\alpha$-regulated PP2A (Fig. 9), which engages PR55 $\alpha$ at three levels of regulation ${ }^{1}$ : inhibition of MOB1-triggered LATS1/2 autoactivation loop (LATS1-S909/LATS2-S872) ${ }^{2}$, destabilizing LATS2 protein and $^{3}$ direct YAP activation (see Figs. 3-7). However, this PR55 $\alpha$-dependent mechanism of YAP activation apparently also activates MST1/2 (see Figs. 3-4), which may be through a feedback mechanism. Thus, the increase in MOB1-T35 phosphorylation in the PR55 $\alpha$-knockdown cells may attribute to the inhibition of the PR55 $\alpha / \mathrm{PP} 2 \mathrm{~A}$ phosphatase activity rather than the increase of MST1/2 kinase activity. Furthermore, MST1/2 level/activity is negatively associated with LATS2 protein stability in both HPNE normal and CD18/HPAF malignant cells in response to PR55 $\alpha$ manipulation, implicating cross-talking or a feedback regulation mechanism among the Hippo pathway components.

PR55 $\alpha$-knockdown results in an increase of LATS2 stability in CD18/HPAF but not in AsPC-1 pancreatic cancer cells (see Fig. 3). Although the exact mechanism causing this difference is unclear, it is likely to be cell-type specific, as the two pancreatic cancer cell lines were originated from different metastatic sites, CD18/ HPAF from the liver and AsPC-1 from ascites ${ }^{39}$. In order to metastasize and recolonize at distant organ sites, primary cancer cells need to adapt and survive a cascade of the environmental challenges by undergoing the processes of invasion $\rightarrow$ intravasation $\rightarrow$ systemic transport $\rightarrow$ extravasation $\rightarrow$ distant colonization ${ }^{50}$. Thus, pancreatic cancer cells metastasizing to the liver versus ascites would have gone through very different adaptive processes and LATS2 stability regulation could be one of those mechanisms needing to be altered to fit different processes. Future studies will be needed to elucidate the mechanism and biological significance of LATS2 regulation during metastasis.

Although PR55 $\alpha$ inhibits the MOB1-triggered LATS1/2 autoactivation that blocks YAP, knockdown of MOB1 in the PR55 $\alpha$-high cells (HPNE-PR55 $\alpha$ or CD18/HPAF) had little effect on YAP level and phosphorylation (Fig. 7a, $Y A P$ and YAP-S127: lanes 3-4 and 5-6). In contrast, knockdown of MOB1 in the PR55 $\alpha$-low cells (HPNE- 


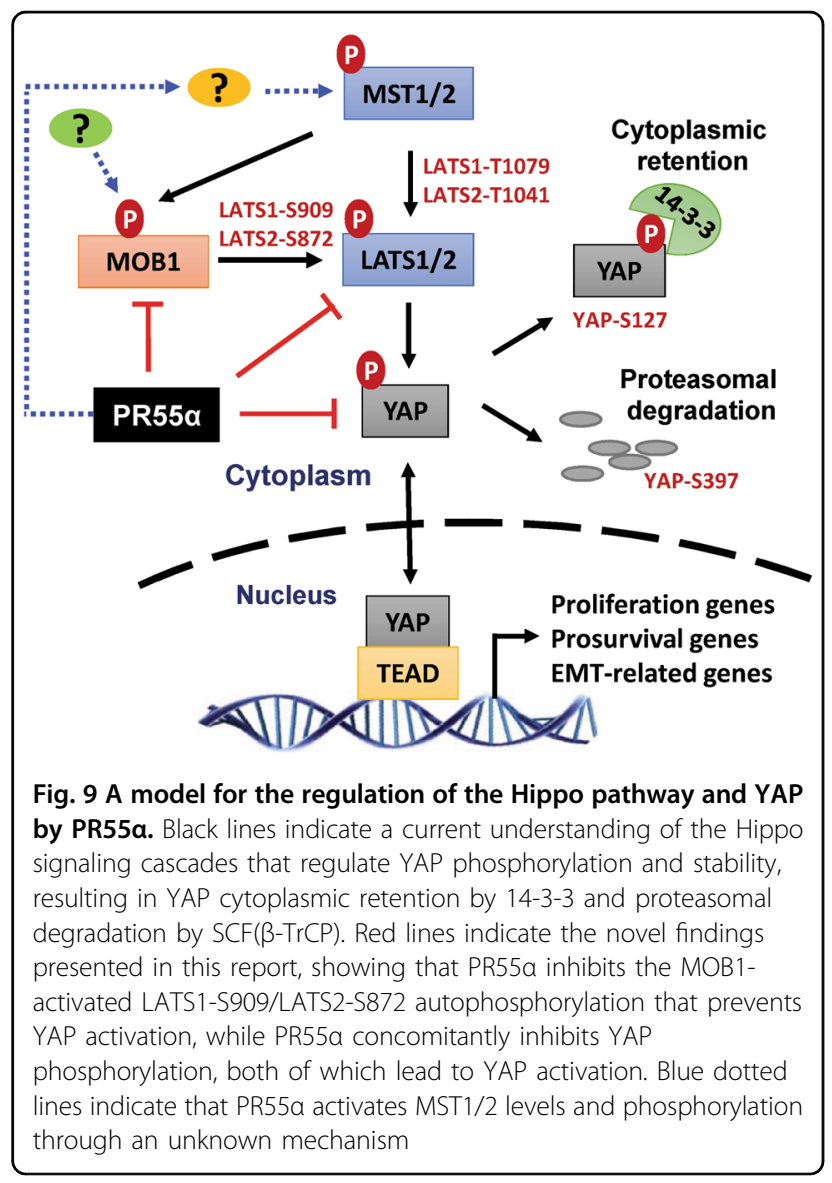

control or CD18/HPAF-PR55 $\alpha$-shRNA) resulted in moderate but noticeable increases in YAP levels (Fig. 7a, YAP: lanes 1-2 and 7-8). Furthermore, knockdown of either LATS1 or LATS2 by siRNA only partially compensated for the loss of PR55 $\alpha$, marginally restoring YAP protein level in the PR55 $\alpha$-knockdown cells (see Fig. 7b, $Y A P$ : lane 6-7 vs. 5). However, such effects are lost when both LATS1 and LATS2 are inhibited by siRNA (see Fig. 7b, YAP: lane 8 vs. lanes 5-7), which suggests there might be an alternative YAP inhibitory pathway whose function is activated by the loss of both LATS1/2 and PR55 $\alpha$ in the cells. Furthermore, the results of Fig. 7 suggest that the role of PR55 $\alpha$ in YAP activation involves both Hippo pathway-dependent and -independent mechanisms, the latter of which could be direct or indirect.

PR55 $\alpha$ directed PP2A activity has been shown to positively regulate several oncogenic pathways that play crucial roles in the oncogenesis of solid tumors, namely the Ras/Raf/MEK, Wnt/ $\beta$-Catenin, and c-Myc signaling pathways ${ }^{17-19,51,52}$. While PR55 $\alpha$ activates the Ras/Raf/ MEK cascade through dephosphorylating KSR-S392 and RaS259/S295 inhibitory sites that block the pathway, its activation of $\beta$-Catenin and c-Myc is via the direct dephosphorylation of $\beta$-catenin-T41/S37/S33 and c-Myc-
T58, respectively, preventing their proteasomal degradation by $\beta$-TrCP. Furthermore, recent studies indicate that cross-talk exists among the PR $55 \alpha$-promoted oncogenic pathways, such as ${ }^{1}$ the Ras/Raf/MEK/ERK signaling that promotes the activation of YAP and c-Myc by increasing their expression ${ }^{2}, \beta$-catenin that synergizes with YAP/ TAZ during cancer progression, and ${ }^{3}$ YAP that is required for KRAS-driving pancreatic tumorigenesis and can compensate for the loss of oncogenic KRAS in the KRAS-addicted pancreatic cells to sustain the malignant phenotypes $^{25-27,53-55}$. These comprehensive data further highlight the significance of PR55 $\alpha$ in tumor promotion and the potential of PR55 $\alpha$ as a therapeutic target for cancer treatment.

\section{Materials and methods \\ Cell culture and treatment}

Human cancer cell lines AsPC-1, Capan-1, CD18/ HPAF, L3.6, HeLa, and SH-SY5Y were obtained from ATCC. HPNE is a line of primary human pancreatic ductal cells immortalized by human telomerase hTERT ${ }^{35}$.

Proteasome inhibitor MG132 (EMD Biosciences) was dissolved in DMSO and cells treated at $10 \mu \mathrm{M}^{56,57}$. Protein synthesis inhibitor CHX (Sigma-Aldrich) was dissolved in water and cells treated at $15 \mu \mathrm{g} / \mathrm{ml}^{58}$.

Cytoplasmic and nuclear extracts were isolated using NE-PER ${ }^{\mathrm{TM}}$ Nuclear and Cytoplasmic Extraction Reagents (Thermo Fisher Scientific). Lamin $\mathrm{A} / \mathrm{C}$ and $\alpha$-tubulin were used as loading controls for nuclear and cytoplasmic extract, respectively ${ }^{59}$.

Additional details of cell culture/treatment are described in Supplementary Materials.

\section{Antibodies}

Antibodies are listed in Supplementary Materials.

\section{Immunoblotting and immunoprecipitation}

Immunoblotting and immunoprecipitation are described in Supplementary Materials ${ }^{20,41,60}$.

\section{Short interfering RNA (siRNA) transfection}

ON-TARGETplus SMARTpool of siRNA duplexes (Dharmacon) were used for silencing LATS1, LATS2, MOB1A, and MOB1B. Control siGENOME nontargeting siRNA (Dharmacon) was designed to target no known genes in human, mouse or rat. The siRNA sequences are described in Supplementary Materials.

Cells were transfected with $100 \mathrm{nmol} / \mathrm{L}$ of siRNA by DharmaFECT-1 (Thermo Fisher Scientific) as instructed by the manufacturer.

\section{shRNA lentiviral vectors and viral infection}

Dox-inducible lentiviral vector (TRIPZ) expressing shRNAs (Dharmacon) were used. shRNA sequences, 
lentiviral production, and viral infection are described in Supplementary Materials.

\section{Retroviral vectors and viral infection}

pRevTet-On retroviral vector (Clontech) expresses the reverse tetracycline-controlled transactivator (rtTA) from the CMV promoter. pRevTRE retroviral vector (Clontech) expresses a gene of interest from the Tet-response element (TRE), which contains seven direct repeats of the tet $O$ operator sequence upstream of a minimal CMV promoter that can be bound by the tTA or rtTA. The pRevTRE-PR55 $\alpha$ retroviral vector contains the PR55 $\alpha$ full-length cDNA sub-cloned from pBluescript-SK(-) vector by HindIII/ClaI digestion.

Flag-YAP and Flag-YAP(5SA) expression vectors were made respectively using plasmids p2xFlag-CMV2-YAP (Addgene \#19045) ${ }^{61}$ and pCMV-flag-YAP-5SA (Addgene \#27371) ${ }^{62}$, both of which encode $\mathrm{N}$ terminally Flag-tagged versions of human YAP (NP_001181973). Coding sequences from both vectors were PCR-amplified using Platinum ${ }^{\mathrm{TM}}-\mathrm{Pfx}$ DNAPolymerase (Thermo Fisher) using forward (5'GTACGCGTCGACAGTGAACCGTCAGAATTGATC

TA-3'; SalI site underlined) and reverse (5'-CATGGAAGATCTCTATAACCATGTAAGAAAG

CTT-3'; BglII site underlined) primers. PCR fragments were then cut with BglII and SalI, gel-purified, and inserted into the BamHI/XhoI sites of pLXSH retroviral vector to produce the final constructs PLXSH-Flag-YAP (WT) and pLXSH-Flag-YAP(5SA). The 5SA mutant carries the following mutations eliminating all LATS1/2phosphorylation sites in YAP: S61A, S109A, S127A/ S128A, S131A, S163A/S164A, and S381A ${ }^{38,42}$.

Retrovirus production and infection are described in Supplementary Materials.

\section{Immunofluorescence and microscopy}

IF and microscopy were performed as described ${ }^{41}$ with additional detail in Supplementary Materials.

Images were taken using a Zeiss-810 confocal laserscanning microscope. Nuclear/cytoplasmic YAP and PR55 $\alpha$ and their co-localization were analyzed by Image $^{63-65}$.

\section{RT-PCR analysis}

Total RNA was isolated using the TRIzol RNAIsolation Reagent (Invitrogen) and analyzed for human ANKRD1, CTGF, CYR61, GAPDH, MOB1A, MOB1B, and Survivin mRNA levels by RT-PCR using the iScript Advanced cDNA Synthesis Kit and SsoAdvanced Universal SYBR Green Supermix (Bio-Rad). The mRNA expressions were normalized with GAPDH-mRNA levels. PCR-primer sequences are listed in Supplementary Materials.

\section{Statistical analysis}

SigmaPlot was used for statistical analyses. Multiple $t$-tests were used for comparison of experimental groups. $P$ values $\leq 0.05$ were considered significant.

\section{Acknowledgements}

We thank Dr Chitra Palanivel for the Flag-YAP/Flag-YAP(5SA) expressing CD18/ HPAF cells, Dr Jixin Dong for the RT-PCR primers for YAP targets, and James Talaska and Janice Taylor for assistance with confocal microscopy (UNMC Microscope Core supported by grant P30GM106397) and Dr Keith Johnson for critical discussions. This work was supported, in parts, by grants R01CA206444 (M.M.O. and S.K.B.), P50CA127297 (S.K.B., M.M.O., and Y.Y.), and a pilot project funded by P30GM106397 (Y.Y. and M.M.O.).

\section{Author details \\ 'Department of Radiation Oncology, University of Nebraska Medical Center, Omaha, NE, USA. ${ }^{2}$ Department of Biochemistry and Molecular Biology, University of Nebraska Medical Center, Omaha, NE, USA. ${ }^{3}$ Department of Internal Medicine, University of Nebraska Medical Center, Omaha, NE, USA}

\section{Conflict of interest}

The authors declare that they have no conflict of interest.

\section{Publisher's note}

Springer Nature remains neutral with regard to jurisdictional claims in published maps and institutional affiliations.

Supplementary Information accompanies this paper at (https://doi.org/ 10.1038/s41389-019-0172-9).

Received: 20 May 2019 Revised: 2 October 2019 Accepted: 4 October 2019 Published online: 28 October 2019

\section{References}

1. Eichhorn, P. J., Creyghton, M. P. \& Bernards, R. Protein phosphatase 2A regulatory subunits and cancer. Biochim. Biophys. Acta 1795, 1-15 (2009).

2. Seshacharyulu, P., Pandey, P., Datta, K. \& Batra, S. K. Phosphatase: PP2A structural importance, regulation and its aberrant expression in cancer. Cancer Lett. 335, 9-18 (2013).

3. Janssens, V., Longin, S. \& Goris, J. PP2A holoenzyme assembly: in cauda venenum (the sting is in the tail). Trends Biochem. Sci. 33, 113 (2008).

4. Ruvolo, P. P. The broken "Off" switch in cancer signaling: PP2A as a regulator of tumorigenesis, drug resistance, and immune surveillance. BBA Clin. 6, 87-99 (2016).

5. Bikel, I. et al. SV40 small t antigen enhances the transformation activity of limiting concentrations of SV40 large T antigen. Cell 48, 321-330 (1987).

6. Pallas, D. C. et al. Polyoma small and middle T antigens and SV40 small t antigen form stable complexes with protein phosphatase 2A. Cell 60, 167-176 (1990).

7. Skoczylas, C., Fahrbach, K. M. \& Rundell, K. Cellular targets of the SV40 small-t antigen in human cell transformation. Cell Cycle 3, 606-610 (2004).

8. Fujiki, H. \& Suganuma, M. Tumor promotion by inhibitors of protein phosphatases 1 and 2A: the okadaic acid class of compounds. Adv. Cancer Res. 61, 143-194 (1993).

9. Mateer, S. C., Fedorov, S. A. \& Mumby, M. C. Identification of structural elements involved in the interaction of simian virus 40 small tumor antigen with protein phosphatase 2A. J. Biol. Chem. 273, 35339-35346 (1998).

10. Nobumori, Y. et al. Characterization of B56Y tumor-associated mutations reveals mechanisms for inactivation of B56y-PP2A. Mol. Cancer Res. 11, 995-1003 (2013).

11. Sablina, A. A., Hector, M., Colpaert, N. \& Hahn, W. C. Identification of PP2A complexes and pathways involved in cell transformation. Cancer Res. 70, 10474-10484 (2010).

12. Yamamoto, $H$. et al. Inhibition of the Wnt signaling pathway by the PR61 subunit of protein phosphatase 2A. J. Biol. Chem. 276, 26875-26882 (2001). 
13. Yang, J. \& Phiel, C. Functions of B56-containing PP2As in major developmental and cancer signaling pathways. Life Sci. 87, 659-666 (2010).

14. Janssens, V. \& Rebollo, A. The role and therapeutic potential of Ser/Thr phosphatase PP2A in apoptotic signalling networks in human cancer cells. Curr. Mol. Med. 12, 268-287 (2012).

15. Zwaenepoel, K., Goris, J., Erneux, C., Parker, P. J. \& Janssens, V. Protein phosphatase 2A PR130/B"alpha1 subunit binds to the SH2 domain-containing inositol polyphosphate 5-phosphatase 2 and prevents epidermal growth factor (EGF)-induced EGF receptor degradation sustaining EGF-mediated signaling. FASEB J. 24, 538-547 (2010).

16. Janssens, V. et al. PP2A binds to the LIM domains of lipoma-preferred partner through its PR130/B" subunit to regulate cell adhesion and migration. J. Cell Sci. 129, 1605-1618 (2016).

17. Adams, D. G. et al. Positive regulation of Raf1-MEK1/2-ERK1/2 signaling by protein serine/threonine phosphatase 2A holoenzymes. J. Biol. Chem. 280, 42644-42654 (2005)

18. Ory, S., Zhou, M., Conrads, T. P., Veenstra, T. D. \& Morrison, D. K. Protein phosphatase 2A positively regulates ras signaling by dephosphorylating KSR1 and Raf-1 on critical 14-3-3 binding sites. Curr. Biol. 13, 1356-1364 (2003).

19. MacDonald, B. T., Tamai, K. \& He, X. Wnt/ $\beta$-catenin signaling: components, mechanisms, and diseases. Dev. Cell 17, 9-26 (2009).

20. Hein, A. L. et al. PR55alpha subunit of protein phosphatase 2A supports the tumorigenic and metastatic potential of pancreatic cancer cells by sustaining hyperactive oncogenic signaling. Cancer Res. 76, 2243-2253 (2016).

21. Sebio, A. \& Lenz, H.-J. Molecular pathways: hippo signaling, a critical tumor suppressor. Clin. Cancer Res. 21, 5002-5007 (2015).

22. Dong, J. et al. Elucidation of a universal size-control mechanism in Drosophila and mammals. Cell 130, 1120-1133 (2007).

23. Pan, D. Hippo signaling in organ size control. Genes Dev. 21, 886-897 (2007)

24. Zanconato, F., Cordenonsi, M. \& Piccolo, S. YAP/TAZ at the roots of cancer. Cancer Cell 29, 783-803 (2016).

25. Kapoor, A. et al. Yap1 activation enables bypass of oncogenic Kras addiction in pancreatic. Cancer Cell 158, 185-197 (2014).

26. Shao, D. D. et al. KRAS and YAP1 converge to regulate EMT and tumor survival. Cell 158, 171-184 (2014).

27. Zhang, W. et al. Downstream of mutant KRAS, the transcription regulator YAP is essential for neoplastic progression to pancreatic ductal adenocarcinoma. Sci. Signal 7, ra42 (2014).

28. Yu, F. X. \& Guan, K. L. The Hippo pathway: regulators and regulations. Genes Dev. 27, 355-371 (2013).

29. Praskova, M., Xia, F. \& Avruch, J. MOBKL1A/MOBKL1B phosphorylation by MST1 and MST2 inhibits cell proliferation. Curr. Biol. 18, 311-321 (2008).

30. Chan, E. H. The Ste20-like kinase Mst2 activates the human large tumor suppressor kinase Lats1. Oncogene 24, 2076-2086 (2005).

31. Ni, L., Zheng, Y., Hara, M., Pan, D. \& Luo, X. Structural basis for Mob1-dependent activation of the core Mst-Lats kinase cascade in Hippo signaling. Genes Dev. 29, 1416-1631 (2015).

32. Ribeiro, P. S. et al. Combined functional genomic and proteomic approaches identify a PP2A complex as a negative regulator of Hippo signaling. Mol. Cell 39, 521-534 (2010).

33. Moreno, C. S., Lane, W. S. \& Pallas, D. C. A mammalian homolog of yeast MOB1 is both a member and a putative substrate of striatin family-protein phosphatase 2A complexes. J. Biol. Chem. 276, 24253-24260 (2001).

34. Guo, C., Zhang, X. \& Pfeifer, G. P. The tumor suppressor RASSF1A prevents dephosphorylation of the mammalian STE20-like kinases MST1 and MST2. J. Biol. Chem. 286, 6253-6261 (2011).

35. Lee, K. M., Nguyen, C., Ulrich, A. B., Pour, P. M. \& Ouellette, M. M. Immortalization with telomerase of the Nestin-positive cells of the human pancreas. Biochem. Biophys. Res. Commun. 301, 1038-1044 (2003).

36. Ma, S., Meng, Z., Chen, R. \& Guan, K. L. The Hippo pathway: biology and pathophysiology. Annu. Rev. Biochem. 88, 577-604 (2019).

37. Meng, Z., Moroishi, T. \& Guan, K. L. Mechanisms of Hippo pathway regulation. Genes Dev. 30, 1-17 (2016)

38. Hergovich, A. Regulation and functions of mammalian LATS/NDR kinases: looking beyond canonical Hippo signalling. Cell Biosci. 3, 32 (2013).

39. Deer, E. L. et al. Phenotype and genotype of pancreatic cancer cell lines. Pancreas 39, 425-435 (2010).
40. Zhu, C. et al. Deubiquitylase USP9X suppresses tumorigenesis by stabilizing large tumor suppressor kinase 2 (LATS2) in the Hippo pathway. J. Biol. Chem. 293, 1178-1191 (2018).

41. Yan, Y. et al. Gamma-irradiation-induced DNA damage checkpoint activation involves feedback regulation between extracellular signal-regulated kinase 1/2 and BRCA1. Cancer Res. 68, 5113-5121 (2008).

42. Zhao, B., Li, L., Tumaneng, K., Wang, C.-Y. \& Guan, K.-L. A coordinated phosphorylation by Lats and CK1 regulates YAP stability through SCF $\beta$-TRCP. Genes Dev. 24, 72-85 (2010).

43. Byles, V. et al. Aberrant cytoplasm localization and protein stability of SIRT1 is regulated by PI3K/IGF-1R signaling in human cancer cells. Int J. Biol. Sci. 6 , 599-612 (2010).

44. Vitulo, N. et al. Characterization and evolution of the cell cycle-associated mob domain-containing proteins in eukaryotes. Evol. Bioinform. 3, 121-158 (2007).

45. Yang, S. et al. Active YAP promotes pancreatic cancer cell motility, invasion and tumorigenesis in a mitotic phosphorylation-dependent manner through LPAR3. Oncotarget 6, 36019-36031 (2015).

46. Chaffer, C. L. \& Weinberg, R. A. How does multistep tumorigenesis really proceed? Cancer Discov. 5, 22-24 (2015).

47. Overholtzer, M. et al. Transforming properties of YAP, a candidate oncogene on the chromosome $11 \mathrm{q} 22$ amplicon. Proc. Natl Acad. Sci. USA 103 12405-12410 (2006).

48. Batra, S. K. et al. Epidermal growth factor ligand-independent, unregulated, cell-transforming potential of a naturally occurring human mutant EGFRvIll gene. Cell Growth Differ. 6, 1251-1259 (1995).

49. Yuan, Y., Li, D., Li, H., Wang, L., Tian, G. \& Dong, Y. YAP overexpression promotes the epithelial-mesenchymal transition and chemoresistance in pancreatic cancer cells. Mol. Med. Rep. 13, 237-242 (2015).

50. Klein, C. A. The metastasis cascade. Science 321, 1785-1787 (2008).

51. Zhang, W. et al. PR55a, a regulatory subunit of PP2A, specifically regulates PP2A-mediated $\beta$-catenin dephosphorylation. J. Biol. Chem. 284, 22649-22656 (2009).

52. Zhang, L. et al. Eya3 partners with PP2A to induce c-Myc stabilization and tumor progression. Nat. Commun. 9, 1047 (2018).

53. Lo Sardo, F., Strano, S. \& Blandino, G. Y. A. P. and TAZ in lung cancer: oncogenic role and clinical targeting. Cancers (Basel) 10, 137 (2018).

54. Huh, D. H., Kim, H. D., Jeong, H.-S. \& Park, W. H. Regulation of TEAD transcription factors in cancer biology. Cells 8, 600 (2019).

55. Muranen, T. et al. ERK and p38 MAPK activities determine sensitivity to PI3K mTOR inhibition via regulation of MYC and YAP. Cancer Res. 76, 7168 (2016).

56. Choudhury, A. D., Xu, H. \& Baer, R. Ubiquitination and proteasomal degradation of the BRCA1 tumor suppressor is regulated during cell cycle progression. J. Biol. Chem. 279, 33909-33918 (2004).

57. Chen, F. et al. Arsenite-induced Cdc25C degradation is through the KEN-box and ubiquitin-proteasome pathway. Proc. Natl Acad. Sci. USA 99, 1990-1995 (2002).

58. Yan, Y., Shay, J. W., Wright, W. E. \& Mumby, M. C. Inhibition of protein phosphatase activity induces p53-dependent apoptosis in the absence of p53 transactivation. J. Biol. Chem. 272, 15220-15226 (1997).

59. Yanagisawa, S. et al. The dynamic shuttling of SIRT1 between cytoplasm and nuclei in bronchial epithelial cells by single and repeated cigarette smoke exposure. PLOS ONE 13, e0193921 (2018).

60. Yan, Y. et al. Protein phosphatase $2 \mathrm{~A}$ has an essential role in the activation of gamma-irradiation-induced G2/M checkpoint response. Oncogene 29, 4317-4329 (2010)

61. Oka, T., Mazack, V. \& Sudol, M. Mst2 and Lats kinases regulate apoptotic function of yes kinase-associated protein (YAP). J. Biol. Chem. 283, 27534-27546 (2008)

62. Zhao, B. et al. Inactivation of YAP oncoprotein by the Hippo pathway is involved in cell contact inhibition and tissue growth control. Genes Dev. 21, 2747-2761 (2007)

63. Schindelin, J. et al. Fiji: an open-source platform for biological-image analysis. Nat. Methods 9, 676-682 (2012).

64. Thomsen, R. \& Christensen, M. H. MolDock: a new technique for high-accuracy molecular docking. J. Med. Chem. 49, 3315-3321 (2006).

65. Xie, S. et al. The endocytic recycling compartment maintains cargo segregation acquired upon exit from the sorting endosome. Mol. Biol. Cell 27, 108-126 (2016). 